Reprod. Nutr. Dévelop., 1987, 27 (2 B), 591-600.

\title{
Influence de l'hormone de croissance sur la production de viande chez les génisses
}

\author{
J. FABRY, V. CLAES, L. RUELLE
}

Centre de Recherches Agronomiques, Station de Zootechnie, Chemin de Liroux, 11, 5800 Gembloux, Belgique.

\section{Summary. Effect of growth hormone on heifer meat production.}

Short-term treatment of bovines with growth hormone $(\mathrm{GH})$ has shown that $\mathrm{GH}$ increases nitrogen balance. In the present experiment we studied the effects of exogenous $\mathrm{GH}$ on weight gain, feed efficiency, carcass grade and meat quality. Twenty heifers of the Belgian White Blue breed were used. After an 8-week pre-experimental period, during which we recorded individual growth rates, the animals were allotted to two groups for 18 weeks ; one group (T) received daily $50 \mu \mathrm{g}$ of pituitary $\mathrm{GH}$ per $\mathrm{kg}$ of liveweight, whereas the other group (C) was given a placebo.

Long-term treatment with $\mathrm{GH}$ induced weight gain (C : $117.1 \pm 25.5 \mathrm{~kg} ; \mathrm{T}: 144.6 \pm$ $23.7 \mathrm{~kg} ; 23.5 \%$ increase, $P<0.05)$ and increased feed efficiency $(C: 10.34 \pm 2.04 \mathrm{~kg}$ $\mathrm{DM} / \mathrm{kg}$ weight gain ; $\mathrm{T}: 8.24 \pm 1.03 \mathrm{~kg} \mathrm{DM} / \mathrm{kg}$ weight gain).

Digestibility of diet dry matter was identical in both groups (C: $60.1 \pm 2.7 \%$; : $59.8 \pm 2.4 \%)$.

There were no difference in the classification of group $C$ and $T$ carcasses according to the EUROP pattern and after monocostal segment dissection (7th rib). Furthermore, the meat analysis of the two groups was identical with respect to protein, fat and collagen content. This was also true for tenderness, $\mathrm{pH}$ and juice loss after cooking.

We concluded that stimulation of bovine weight gain by $\mathrm{GH}$ was significant and efficient while meat quality and carcass grade were not improved.

\section{Introduction.}

Pour la race Blanc Bleu Belge, en particulier, les sélectionneurs ont considérablement contribué à l'amélioration des performances zootechniques des animaux destinés à la production dé viande : vitesse de croissance, indice de consommation et qualité de la carcasse. Néanmoins, certaines modifications de l'état hormonal de cette population d'élite peuvent encore améliorer, voire optimaliser, ces paramètres de production.

L'hormone de croissance $(\mathrm{GH})$ ainsi que d'autres hormones qui s'y rattachent (somatomédines, somatocrinine), ont suscité un important courant d'intérêt ces dernières années, en temps que stimulateurs de croissance. Cependant, les premières expérimentations consacrées à l'étude des effets de la $\mathrm{GH}$ exogène sur la croissance se sont souvent soldées par des échecs chez le porc, le mouton et le bovin (Turman et Andrews, 1955 ; Henricson et Ullberg, 1960 ; Wheatley, 1966 ; 
Vézinhet, 1973 ; Reklewska, 1974 ; Muir et al., 1983 ; Brumby, 1959 ; Znidar, 1976).

La GH isolée de l'hypophyse est un produit rare et onéreux qui, à l'époque, faute de techniques de purification adéquates, contenait très vraisemblablement des quantités appréciables d'impuretés (TSH, PRL) susceptibles de modifier son activité anabolique. D'autres facteurs peuvent aussi intervenir, comme l'utilisation d'une hormone de croissance non spécifique à l'espèce qui induit une réaction immunitaire et neutralise toute action anabolique. Le mode d'administration de I'hormone et la dose utilisée ont également une importance majeure (Fabry, résultats non publiés). De plus, si l'on veut mettre en évidence une différence statistique il faut nécessairement procéder à des expérimentations d'une durée suffisante avec un nombre élevé d'animaux.

Pour le porc, les travaux de Machlin (1972) et de Chung et al. (1985) montrent que I'injection quotidienne de pGH améliore significativement le taux de croissance en augmentant la masse musculaire ainsi que la taille du cartilage épiphysaire.

Wagner et Veenhuizen (1978) signalent une élévation importante du croît journalier chez l'agneau qui reçoit une injection quotidienne d'hormone de croissance durant 14 jours. Les différences observées ne sont cependant pas significatives en raison du faible nombre d'animaux expérimentaux. Pour Muir et al. (1983) l'effet bénéfique de l'hormone de croissance se situe essentiellement au niveau de I'indice de consommation. Chez le taureau, le mélange diethylstilboestrol $+\mathrm{bGH}$ améliore le croît journalier de 12 à $13 \%$ (Zhuravel, 1972). Par contre, pour Brumby (1959) l'administration de $0,10 \mathrm{mg}$ de $\mathrm{bGH} / \mathrm{kg} \mathrm{PV} / \mathrm{j}$ ne modifie pas la croissance des génisses $(+3 \%$, NS). Chez le bœuf, I'association bGH $+17 \beta$ œstradiol s'avère plus efficace que la bGH ou le $17 \beta$-œstradiol utilisé seul. Selon Roche (1985) l'effet anabolique de ce dernier serait supérieur à celui de la bGH.

A I'heure actuelle, aucune étude n'a pu démontrer l'effet d'une administration à long terme d'hormone de croissance sur la production de viande bovine. Afin de combler cette lacune, nous avons procédé à une expérimentation de dixhuit semaines sur un nombre élevé de génisses de race Blan Bleu Belge (BBB) en croissance-engraissement.

\section{Matériel et méthodes.}

L'essai porte sur vingt génisses de la race BBB achetées, à la sortie de l'herbage, à l'âge de 15 mois. Hormis leur bon état sanitaire et leur appartenance au rameau mixte de la race (BBB-m), ces animaux ne répondent à aucun critère particulier. Ils reflètent la qualité moyenne des génisses disponibles en automne pour la production de viande et pèsent, en moyenne, $397 \pm 30 \mathrm{~kg}$.

Les animaux sont maintenus en stabulation entravée, sur litière de paille, ce qui permet de contrôler les consommations alimentaires individuelles et de fournir une ration adaptée à chaque animal.

L'abreuvement est accessible à volonté et la litière renouvelée deux fois par semaine afin d'assurer aux animaux un maximum de confort et d'hygiène. 
La ration, composée d'un aliment concentré du commerce, dosant $14 \%$ de protéines totales est apportée deux fois par jour, à $8 \mathrm{~h}$ et à $15 \mathrm{~h}$. Ce concentré est distribué à volonté et complémenté par un complexe minéralo-vitaminé à raison de $100 \mathrm{~g} /$ jour/animal. Le bon fonctionnement du rumen est assuré par l'ingestion quotidienne de $\pm 1 \mathrm{~kg}$ de paille.

La bGH utilisée, extraite à partir d'hypophyses prélevées sur bovins au moment de l'abattage, est purifiée par Intervet au moyen de techniques adéquates (Boxmeer, Holland). La présence d'éventuels contaminants TSH, LH et PRL n'a pu être mise en évidence au moyen des systèmes radioimmunologiques classiques. L'activité biologique de la $\mathrm{GH}$ est de $1 \mathrm{Ul} / \mathrm{mg}$.

Les animaux du lot traité reçoivent, quotidiennement, par injection souscutanée réalisée au niveau de l'épaule, l'hormone de croissance ( $50 \mu \mathrm{g} / \mathrm{kg}$ PV) préalablement dissoute dans $2,5 \mathrm{ml}$ de tampon bicarbonate $(\mathrm{pH}=10,2)$. Aux animaux témoins, on administre un placebo constitué par 2,5 $\mathrm{ml}$ du même tampon. Les modalités d'injection de l'hormone ainsi que la dose utilisée ont fait l'objet d'une mise au point préalable.

L'essai est divisé en trois périodes successives : une première partie préexpérimentale de 45 jours (S-6 à $S$ 0), la période expérimentale proprement dite de 126 jours ( $\mathrm{S} 0$ à $S 18$ ) et finalement une période post-expérimentale de 10 jours avant l'abattage des animaux (S 19). La mesure de la digestibilité de la ration a eu lieu aux semaines 7 et 8 de l'essai ; tandis que l'abattage a été réalisé fin de la semaine 19.

Les génisses sont réparties en deux lots, selon les poids vifs, les gains quotidiens réalisés au cours de la phase pré-expérimentale et en tenant compte de la conformation des animaux : le lot témoin (T) et le lot traité $(\mathrm{GH})$.

Les animaux sont pesés toutes les quinzaines à la même heure $(8 \mathrm{~h})$. La pesée est faite deux jours consécutifs au début (S 0) et en fin d'essai (S 18). La quantité de $\mathrm{MS}$ ingérée est calculée pour chaque animal et par quinzaine.

La technique à l'oxyde de chrome $\left(\mathrm{Cr}_{2} \mathrm{O}_{3}\right)$ a été choisie pour mesurer la digestibilité apparente de la ration. Comme l'ont montré Thill et al. (1978) l'oxyde de chrome, marqueur externe de la phase solide des digesta, n'est pas absorbé. De plus, on sait que son comportement est semblable à celui du radiocérium, considéré, par de nombreux auteurs, comme marqueur idéal (McRae, 1974). Deux méthodes ont été utilisées pour apprécier la qualité des carcasses des génisses : la grille communautaire de classement des carcasses de gros bovins EUROP (prévue par les règlements de la CEE $\mathrm{n}^{\circ} 1208-81$ et 2930-81) et l'analyse de la composition d'un segment costal prélevé au niveau de la $7^{\mathrm{e}}$ côte. Celui-ci est disséqué en ses tissus principaux : osseux, musculaire, adipeux, sous-cutané, intermusculaire et conjonctif. Les muscles sont ensuite individualisés et pesés.

\section{Résultats.}

1. Gain quotidien moyen (GQM).

Période pré-expérimentale. - Au cours du pré-essai, les animaux du lot témoin ont gagné, en moyenne, $43,3 \mathrm{~kg}$ de poids vif, ce qui correspond à un 
GQM de $0,96 \mathrm{~kg} / \mathrm{j}$. Les animaux du futur lot traité ont réalisé un gain total de $42,2 \mathrm{~kg}$, c'est-à-dire un GQM de $0,94 \mathrm{~kg} / \mathrm{j}$. Les GQM observés ne sont pas différents du niveau $\alpha=0,1$.

Période expérimentale. - Pendant la phase expérimentale, l'influence de la bGH sur le gain de poids a été très importante et significative : $\pm 220 \mathrm{~g} / \mathrm{j}$ anim. soit $+23,5 \%$ par rapport au lot témoin.

L'effet de l'anabolisation se marque dès la première pesée : après quatorze jours de traitement, les animaux du lot $\mathrm{GH}$ ont déjà gagné $5 \mathrm{~kg}$ de plus que les témoins $(+50 \%, N S)$. La différence de gain devient significative entre la quatrième et la sixième semaine $(+47 \% \mathrm{P}<0,05)$. A ce moment, $61 \%$ de l'écart final du gain de poids vif entre les deux lots sont acquis (fig. 1).

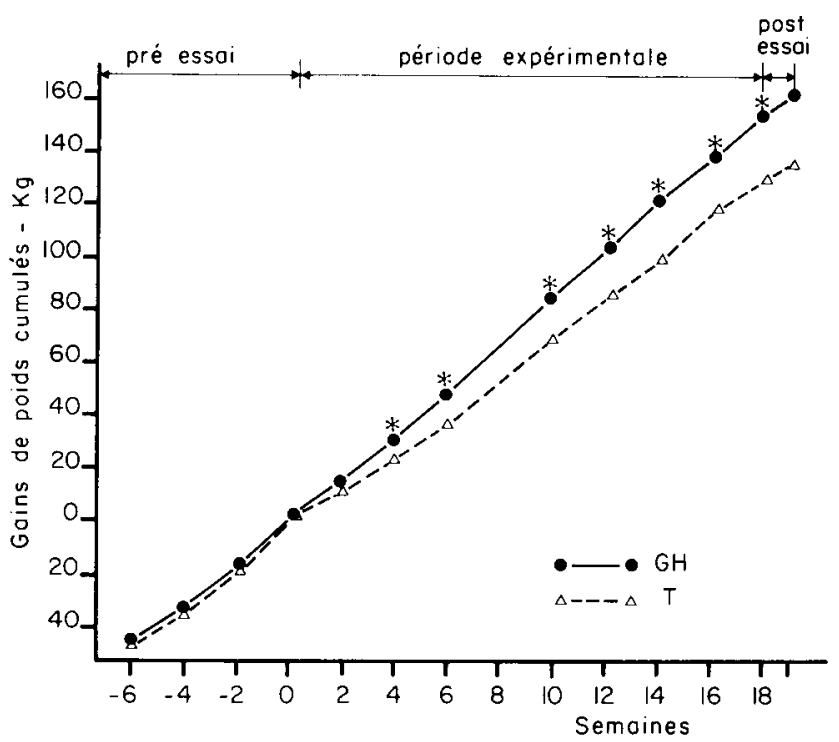

FIG. 1. - Evolution des gains de poids cumulés au cours des périodes pré-expérimentale, expérimentales et post-expérimentale $\left(^{*} \mathrm{P}<0,05\right)$.

Si on fait abstraction de la période durant laquelle les animaux ont été perturbés par les mesures de digestibilité (S4-S8) on obtient les GQM suivants: $1,19 \mathrm{~kg} / \mathrm{j}$ pour le lot anabolisé et $0,91 \mathrm{~kg} / \mathrm{j}$ pour le lot témoin, soit une augmentation significative de $280 \mathrm{~g} / \mathrm{j}(+31 \%, \mathrm{P}<0,001)$.

L'action de la bGH s'installe rapidement et se maintient tout au long de l'essai. Sur l'ensemble de la phase expérimentale, les poids moyens $(Y)$ évoluent linéairement en fonction du temps $(X)$ (lot $T: Y=0,94 X+434,18, r=0,99$ et lot $\mathrm{GH}: \mathrm{Y}=1,15 \mathrm{X}+436,72, r=0,99)$.

Période post-expérimentale. - Dès la fin des injections de $\mathrm{GH}$, le croît journalier des animaux anabolisés chute ( $73 \%$ de ce qu'il était pendant le traitement) et devient similaire à celui des témoins $(0,84 \pm 0,60 \mathrm{~kg} / \mathrm{j}$ contre $0,89 \pm$ $0,81 \mathrm{~kg} / \mathrm{j})$. 
2. Consommation.

A aucun moment de l'essai nous n'avons observé une différence entre les ingestions quotidiennes des deux lots. Les consommations journalières sont cependant légèrement inférieures pour les animaux témoins (différence $140 \mathrm{~g} / \mathrm{j}$; $+1,5 \%$; NS). Toutefois, si l'on rapporte ces valeurs au poids moyen des animaux, la situation s'inverse : l'ingestion des témoins est légèrement supérieure à celle des traités $(+2 \%, N S)$. Les ingestions exprimées en fonction du poids métabolique des animaux $\left(P^{0,75}\right)$ diffèrent de moins $d^{\prime}$ un pourcent entre les lots.

\section{Digestibilité.}

La bGH n'a exercé aucune influence significative sur la digestibilité apparente de la matière sèche. Celle-ci est de $59,8 \pm 2,4 \%$ pour le lot $\mathrm{GH}$ et de $60,1 \pm$ $2,7 \%$ pour le lot $\mathrm{T}(-0,5 \%, \mathrm{NS})$ (tabl. 1$)$.

\section{Efficacité alimentaire.}

La relation entre les indices de consommation ( $\mathrm{lc}=\mathrm{MS}$ ingérée en $\mathrm{kg}$ par $\mathrm{kg}$ de croît! et les GQM ( $\mathrm{kg} / \mathrm{j}$ ) est de type curvilinéaire (Lambot et al., 1983). Les relations sont les suivantes :

$$
\begin{aligned}
& \text { lc }=21,84(0,45)^{\mathrm{GOM}},(r=0,90) \text { pour le lot } \mathrm{T} \text {, et } \\
& \text { lc }=10,57(0,78)^{\mathrm{GOM}},(\mathrm{r}=0,80) \text { pour le lot } \mathrm{GH} \text {. }
\end{aligned}
$$

On constate que les animaux traités par $\mathrm{GH}$ se révèlent beaucoup plus efficaces dans la transformation des aliments en viande. En effet, pendant la phase expérimentale, nous avons enregistré une différence de $2,10 \mathrm{~kg}$ d'aliments (MS) par kg de gain en faveur des génisses anabolisées (tabl. 1).

TABLEAU 1

Performances zootechniques au cours de la période expérimentale

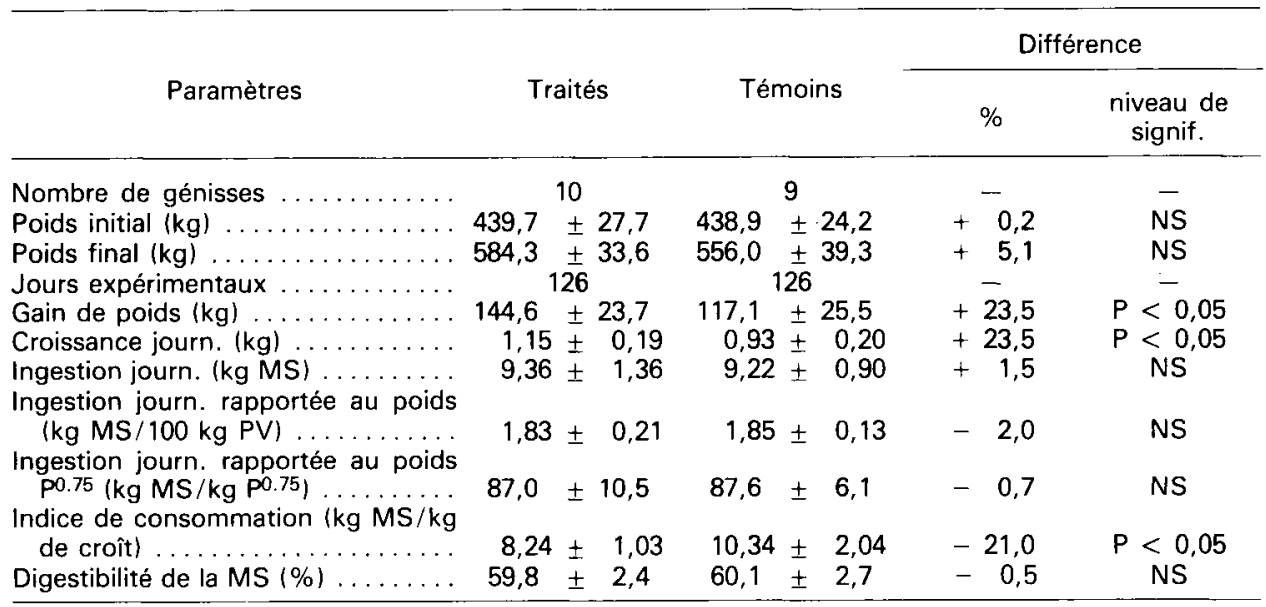

NS : non significatif. 


\section{Données à l'abattage.}

Le poids des carcasses des animaux du lot $\mathrm{GH}$ ne diffère pas significativement de celui des animaux du lot $T$. La relation entre le poids de carcasse $(Y)$ et le poids vif final $(X)$ est très étroite, tant pour le lot traité $(Y=0,548 X+18,579$, $r=0,93, P<0,001)$ que pour le lot témoin $(Y=0,617 X-15,450, r=0,98$, $P<0,001)$.

Le rendement à l'abattage pour les sujets témoins est légèrement supérieur à celui des traités par l'hormone de croissance; l'écart de 1,7\% est significatif au niveau 0,1 . Le rendement carcasse commercial est de $57,5 \%$ pour le lot $T$ et de $56,5 \%$ pour le lot $\mathrm{GH}(-1 \%$, NS).

Les carcasses ont été classées selon la grille pour gros bovins (EUROP). Dans cette grille, on les retrouve dans les classes $R_{3}, R_{4}, O_{3}$ et $O_{4}$. Aucune différence n'est observée à ce niveau.

Les résultats de la dissection des segments monocostaux montrent que les carcasses des sujets traités contiennent plus de muscle et moins de graisse (tabl. 2). Les rapports muscles totaux/os et muscles totaux/tissus conjonctivoadipeux sont un peu plus élevés dans le lot GH sans toutefois être significativement différents.

\section{TABLEAU 2}

Données à l'abattage

\begin{tabular}{|c|c|c|c|c|}
\hline \multirow[b]{2}{*}{ Paramètres } & \multirow[b]{2}{*}{ Traités } & \multirow[b]{2}{*}{ Témoins } & \multicolumn{2}{|c|}{ Différence } \\
\hline & & & $\%$ & $\begin{array}{l}\text { niveau de } \\
\text { signif. }\end{array}$ \\
\hline $\begin{array}{l}\text { Poids de la carcasse }(\mathrm{kg}) \ldots \ldots \ldots \\
\text { Rendement à l'abattage }(\%) \ldots \ldots \\
\text { Composition du monocostal } 7(\%)\end{array}$ & $\begin{array}{c}334 \pm 21 \\
57,9 \pm 1,2\end{array}$ & $\begin{array}{c}324 \pm 25 \\
58,9 \pm 0,9\end{array}$ & $\begin{array}{l}+3 \\
-1,7\end{array}$ & $\begin{array}{l}\text { NS } \\
\text { NS }\end{array}$ \\
\hline 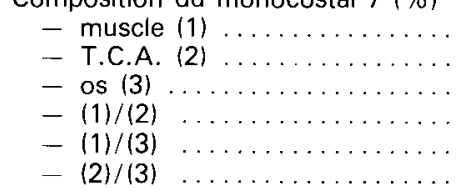 & $\begin{array}{r}55,1 \pm 3,9 \\
28,0 \pm 3,9 \\
16,9 \pm 1,5 \\
2,0 \pm 0,4 \\
3,3 \pm 0,4 \\
1,7 \pm 0,3\end{array}$ & $\begin{array}{r}54,0 \pm 3,0 \\
28,9 \pm 2,7 \\
17,0 \pm 1,5 \\
1,9 \pm 0,2 \\
3,2 \pm 0,4 \\
1,7 \pm 0,2\end{array}$ & $\begin{array}{l}+2,0 \\
-3,1 \\
-0,6 \\
+5,3 \\
+3,1 \\
-\end{array}$ & $\begin{array}{l}\text { NS } \\
\text { NS } \\
\text { NS } \\
\text { NS } \\
\text { NS } \\
\text { NS }\end{array}$ \\
\hline
\end{tabular}

T.C.A. : tissu conjonctivo-adipeux. NS : non significatif.

\section{Qualité de la viande.}

Les principales caractéristiques de la viande des animaux expérimentaux sont décrites ailleurs (Adam et al., 1985). Un bref résumé est repris ci-dessous. Les $\mathrm{pH}$ mesurés sur les carcasses et sur le segment monocostal 7 sont identiques chez les animaux des deux lots. Quelle que soit la méthode de détermination utilisée (four à micro-ondes ou cuisson à la vapeur humide), il n'y a pas de différence entre les pertes de jus de la viande des animaux traités et témoins. Les résultats obtenus par les cinq méthodes utilisées pour apprécier la tendreté de la viande montrent que celle-ci est d'une tendreté très moyenne pour tous les animaux (Adam 
et al., 1985). La localisation des dépôts graisseux est très variable d'un sujet à l'autre; par contre, la viande est uniformément marbrée. Le pourcentage de graisse est toujours élevé ; il représente de 7 à $8 \%$ du poids de la viande fraîche et influence sensiblement le niveau de classement des carcasses. La teneur en collagène est faible et correspond au niveau moyen que I'on rencontre dans le bétail viandeux de la race Blanc Bleu Belge.

\section{Discussion.}

L'accélération de la croissance que nous avons obtenue avec l'hormone de croissance est supérieure à celle mentionnée dans la littérature pour d'autres espèces. Elle correspond aux améliorations que I'on peut obtenir chez la génisse, avec les anabolisants classiques (Galbraith, 1980 ; Heitzman et Chan, 1974).

L'effet de la bGH sur le gain de poids quotidien se marque après 2 semaines de traitement. Toutefois, la différence de gain entre animaux appartenant au lot témoin ou au lot traité ne devient significative qu'entre la quatrième et la sixième semaine. Par la suite, elle se maintient à ce niveau de signification jusqu'à la fin de l'essai.

Si $Y$ est égal au GQM $(\mathrm{kg} / \mathrm{j}$, animaux répartis en classes en fonction de leur gain de poids journalier), des sujets traités et $X$ à celui des témoins, on a les relations suivantes : $Y=0,726 X+0,477(r=0,99)$ et $100 Y / X(\%)=72,6+47,7 X$ $(\mathrm{kg} / \mathrm{j})$ ce qui indique que l'amélioration apportée par la bGH n'est pas indépendante du niveau des gains des témoins. Ainsi, pour des GQM variant de 0,8 à $1,2 \mathrm{~kg}$ par tranche de $0,1 \mathrm{~kg}$, l'amélioration exprimée en $\%$ du gain des témoins serait égale à $32 \%$ (ou $+246 \mathrm{~g}), 26 \%(+234 \mathrm{~g}), 20 \%(+200 \mathrm{~g})$ et $16 \%$ $(+176 \mathrm{~g})$. Dès l'arrêt des injections d'hormone la différence dans le rythme du gain de poids des deux lots s'estompe rapidement. Ceci tend à démontrer que les effets bénéfiques de la $\mathrm{GH}$ ne sont maintenus que si les taux circulants d'hormone sont élevés.

La consommation quotidienne des animaux traités est légèrement supérieure à celle des animaux témoins. Les régressions gain de poids vif $(X)-$ quantité de MS consommée $(Y)(Y=7,97 X+17,26, r=0,99$ pour le lot $\mathrm{GH}$ et $Y=9,25 X$ $+75,60, r=1,00$ pour le lot témoin) montrent, qu'à ingestion égale, les animaux traités réalisent des gains de poids supérieurs. Les consommations exprimées en fonction du poids métabolique $\left(\mathrm{P}^{0,75}\right)$ sont identiques dans les deux lots.

Des résultats analogues ont été signalés chez le porc (Chung et al., 1985), l'agneau (Muir et al., 1983) et la vache laitière (Peel et al., 1983 ; Bauman et al., 1982). Par contre, pour Bauman et al. (1984) le traitement à long terme (188 jours) des vaches laitières avec la bGH augmente l'ingestion d'environ $15 \%(P<0,05)$. Selon ces auteurs, les animaux adapteraient leur ingestion alimentaire pour pouvoir produire un surcroît de lait. D'autre part, Peel et al. (1983) rapportent qu'en fin de lactation, la bGH $(51,5 \mathrm{UI} / \mathrm{j})$ provoque une réduction significative de la consommation $(-16 \%, P<0,05)$. Néanmoins, après 126 jours d'expérimentation avec les génisses à l'engrais, nous n'enregistrons aucune modification significative des consommations alimentaires.

La diminution des indices de consommation (Ic) du lot traité est étroitement liée à l'augmentation du GQM puisque la consommation globale dans chacun des 
lots est similaire. Les régressions curvilinéaires Ic (Y, $\mathrm{kg} \mathrm{MS/kg} \mathrm{de} \mathrm{croît)} \mathrm{-} \mathrm{GQM}$ $(X, \mathrm{~kg} / \mathrm{j})$ montrent que pour un même gain quotidien, l'efficacité alimentaire est supérieure chez les animaux traités $\left(-21 \% ; Y=21,84(0,45)^{X}, r=0,90\right.$ pour le lot $T$ et $Y=10,57(0,78)^{x}, r=0,80$ pour le lot $\left.\mathrm{GH}\right)$. Chez le porc, la $\mathrm{pGH}$ améliore aussi l'efficacité alimentaire de $13 \%$ ou de $4 \%$ selon l'essai (Chung et al., 1985). Chez I'agneau, l'injection quotidienne de bGH entraîne une réduction de l'Ic de 7,4 \% (Muir et al., 1983) à $16 \%$ (Wagner et Veenhuizen, 1978). Par contre, d'autres expériences n'ont pas révélé de modification de cet indice (Brumby, 1959 ; Car et al., 1976). Nos résultats sont assez proches de ceux obtenus avec les anabolisants classiques ou les améliorations de Ic atteignent $20 \%$ chez le veau (Vanderwal et al., 1975), et $40 \%$ chez la génisse (Chan et al., 1975) ou la vache de réforme (Galbraith, 1980). Pour le taurillon Blanc Bleu Belge, I'amélioration avoisine $30 \%$ chez l'animal mixte et $15 \%$ chez le culard (Lambot et al., 1982, 1983).

L'apport de bGH exogène n'affecte pas la digestibilité de la matière sèche et de l'azote chez la vache laitière (Peel et al., 1981 ; Eisemann et al., 1984). Les résultats que nous avons obtenus avec la $\mathrm{GH}$ corroborent parfaitement ceux qui découlent de l'utilisation des anabolisants classiques, à savoir que la stimulation de la croissance ne résulte pas d'une amélioration de la digestibilité de la ration. La $\mathrm{GH}$ semble donc agir au niveau de l'utilisation des nutriments, après leur résorption intestinale.

L'influence de la $\mathrm{GH}$ exogène sur le poids des carcasses et sur le rendement à l'abattage n'est pas significative. La relation qui unit le poids de la carcasse (Y) au poids vif final $(X)(Y=0,617 X-15,45, r=0,98$ pour le lot $T, Y=0,548 X+$ $18,579, r=0,93$ pour le lot $\mathrm{GH}$ ) montre cependant que pour un même poids vif les carcasses sont en moyenne plus lourdes chez les animaux témoins. La diminution du rendement à l'abattage résulte d'une augmentation de la masse du cinquième quartier. Le poids des reins et celui de la rate sont en effet plus élevés chez les animaux auxquels on injecte la bGH (respectivement $+30 \%, \mathrm{P}<0,02$ et $+9,2 \%$ ), NS). Une telle " viscéromégalie " a également été signalée par Muir et al. (1983) chez l'agneau et par Chung et al. (1985) chez le porc.

Le classement des carcasses selon la grille EUROP n'a révélé aucune différence entre les deux lots. Chez les animaux anabolisés, la dissection du segment monocostal $n^{\circ} 7$ fait apparaître une proportion de muscles largement plus élevée et un pourcentage de graisse apparemment plus bas. Ces observations confortent celles réalisées chez le mouton (Muir et al., 1983 ; Wagner et Veenhuizen, 1978) et le porc (Machlin, 1972) sans pour autant en atteindre l'ampleur. II y a cependant loin d'y avoir unanimité quant au rôle lipolytique de l'hormone de croissance chez les animaux domestiques. Toutefois, avant de tirer toute conclusion définitive, il $y$ aurait lieu d'uniformiser, voire de standardiser les méthodes d'estimation de la graisse à la fois au niveau de la carcasse et de la viande. Dans notre étude, chacune des trois méthodes utilisées pour évaluer les carcasses montre qu'il n'y a pas d'effet lipolytique significatif de I'hormone de croissance chez le bovin. Pour Duquette et al. (1983) l'effet lipolytique de GH ne semble pas évident même in vitro. Ajoutons que la composition en acides gras supérieurs de la graisse périrénale n'a pas été 
modifiée par le traitement à l'hormone de croissance si ce n'est pour l'acide myristique $(+0,8 \%, P<0,04)$.

Pour les animaux de notre essai, Adam et al. (1985) n'ont observé aucun changement significatif de la qualité de la viande : $\mathrm{pH}$, jutosité, teneur en eau, tendreté et composition macroscopique. A notre connaissance, l'incidence de la bGH sur la qualité de la viande des génisses n'a jamais été évaluée. Les quelques auteurs qui ont étudié l'influence de l'anabolisation sur les principales qualités de la viande n'ont relevé que de légères modifications au niveau des pertes de jus à la cuisson et de la tendreté de la viande (Grandadam et al., 1975).

En conclusion, il apparaît que la bGH exerce une influence significative sur les performances zootechniques des génisses en croissance engraissement, en permettant une accélération importante de la croissance avec une amélioration substantielle de l'efficacité alimentaire.

$12^{e}$ Réunion du groupe Développement INRA, Montpellier, 28-30 mai 1986.

\section{Références}

ADAM J. J., FABRY J., ETTAIB A., DEROANNE C., 1985. Effet d'un apport d'hormone de croissance bovine sur la qualité des viandes de génisse blanc-bleu-belge. Rec. Méd. vét., 85. 655-663.

BAUMAN D. E., DE GEeTER M. J., PEEL C. J., LANZA G. M., GOREWIT R. C., HAMMOND R. W., 1982. Effect of recombinantly-derived bovine growth hormone $\langle\mathrm{bGH}\rangle$ on lactational performance of high-yielding dairy cows. J. Dairy Sci., 65 (Suppl. 1), p. 121.

BAUMAN D. E., Mc CUTCHEON S. N., 1984. The effects of growth hormone and prolactin on metabolism. In A. DOBSON. Proc. VI int. Symp. on Ruminant physiology. Reston Publ. Co. Inc. (in press).

BRUMBY P. J., 1959. The influence of growth hormone on growth in young cattle. New Zealand J. agr. Res., 2, 683-689.

CAR M., ZNIDAR A., FILIPAN T., 1976. An effect of the treatment of young steers with STH (growth hormone) upon nitrogen retention in intensive feeding. Poljopr. Znan Snotra., 37. p. 183.

CHAN K. H., HEITZMAN R. J., KITCHANHAM B. A., 1975. Digestibility and N-balance studies on growing heifers implanted with trenbolone acetate. Br. vet. J., 131, 170-174.

CHUNG C. S., ETHERTON T. D., WIGGINS J. P., 1985. Stimulation of swine growth by porcine growth hormone. J. anim. Sci., 60, 118-130.

DUQUETTE P. F., MUIR L. A., SCANES C. G., 1983. Growth hormone doesn't cause lipolysis of ovine adipose tissue in vitro. J. anim. Sci, 57 (suppl. 1), p. 192.

EISEMANN J. H., TYRRREL $H$. F., HAMMOND A. C., REYNOLDS P. J., BAUMAN D. E, HAALAND G. L., VARGA G. H., 1984. Influence of bovine growth hormone on energy and nitrogen balance in Hereford heifers. J. anim. Sci, 59 (suppl. 1), p. 201.

GALBRAITH H., 1980. The effect of trenbolone acetate on growth, blood hormones and metabolites, and nitrogen balance of beef heifers. Anim. Prod., 30, 389-394.

GRANDADAM J. A., SCHEID J. P., DREUX H., DEROY R., 1975. Influence de différentes préparations anabolisantes sur la qualité de la viande du veau. Rec. Méd. vét., 151, 355-362.

HEITZMAN R. J., CHAN K. H., 1974. Alterations in weight gain and levels of plasma metabolites, proteins, insulin and free fatty acids following implantation of an anabolic steroid in heifers. Br. Vet. J, 130, 532-537.

HENRICSON B., ULLBERG S., 1960. Effect of pig growth hormone on pigs. J. anim. Sci., 19. $1002-1012$. 
LAMBOT O., VAN EENAEME C., BIENFAIT J. M., GIELEN M., ISTASSE L., 1982. Effet du trenbolone associé au $17 \beta$-œstradiol sur des taurillons des types culard et mixte en croissance-engraissement. I. Croissance et efficience alimentaire. Ann. Méd. vét., 126, 477-491.

LAMBOT O., VAN EENAEME C., GIELEN M., BIENFAIT J. M., 1983. Effets des associations anabolisantes trenbolone $+17 \beta$-œstradiol, progestérone $+17 \beta$-œestradiol et du zéranol chez les jeunes taureaux en croissance-engraissement. Revue de l'Agriculture, 36, 5-27.

MACHLIN L. J., 1972. Effect of porcine growth hormone on growth and carcass composition of the pig. J. anim. Sci., 35, 794-800.

Mc RAE J. C., 1974. The use of intestinal marker to measure digestion function in ruminants. Proc. Nut. Soc., 33, 147-154.

MUIR L. A., WIENS S., DUQUETTE P. F., RICKES E. L., 1983. Effects of exogenous growth hormone and diethylstilbestrol on growth carcass composition of growing lambs. J.anim. Sci., 56, 1315-1323.

PEEL C. J., BAUMAN D. E., GOREWIT R. C., SNIFFEN C. J., 1981. Effect of exogenous growth hormone on lactational performance in high yielding dairy cows. J. Nutr., 111, 1662-1671.

PEEL C. J., FRANK T. J., BAUMAN D. E., GOREWIT R. C., 1983. Effect of exogenous growth hormone in early and late lactation on lactational performance of dairy cows. J. Dairy Sci., 66, 776-782.

REKLEWSKA B., 1974. A note on the effect of bovine somatotrophic hormone on wool production in growing lambs. Anim. Prod., 19, p. 253.

ROCHE J. F., 1985. The effects of steroid hormones and xenobiotics on growth of farm animals, 39-51. In P. J. BUTTERY, N. B. HAYNES, D. B. LINDSAY. Control and manipulation of growth, Butterworth London, 1986.

THILL N., FRANÇOIS E., THEWWIS A., THIELEMANS M. F., 1978. Comparaison chez le mouton de l'oxyde de chrome papier et du radiocérium, marqueurs de la phase solide des digesta. Ann. Zootech., 27, 363-376.

TURNAN E. J., ANDREWS F. N., 1955. Some effects of purified anterior pituitary growth hormone on swine. J. anim. Sci., 14, 7-18.

VANDERWAL P., BERENDE P. L. M., SPRIETSMA J. E., 1975. Effect of anabolic agents on performances of calves. J. anim. Sci., 41, 978-985.

VEZINHET A., 1973. Influence de I'hypophysectomie et de traitements à l'hormone somatotrope bovine sur la croissance relative de l'agneau. Ann. Biol. Anim. Bioch. Biophys., 13, 51-73.

WAGNER J. F., VEENHUIZEN E. L., 1978. Growth performance, carcass composition and plasma hormone levels in wether lambs when treated with growth hormone and thyroprotein. J. anim. Sci., 46 (suppl. 1), p. 397.

WHEATLEY I. S., WALLACE A. L. C., BASSETT J. M. 1966 . Metabolic effects of ovine growth hormone in sheep. J. Endocrinol., 35, 341-353.

ZHURAVEL A. A., 1972. The use of hormonal preparations for increasing productivity of livestock. Primenenie biogen stimulyatorov $v$ zhivotnovodstve i izuch. Mekhanizma ikh Deistviya Borovsk USSR, p. 135.

ZNIDAR A., 1976. Effect of growth hormone on weight gain and feed conversion of simmental bull calves. Poljoprivredna Znenstrna Smotra, 47, p. 171-176. 